\title{
Components of Purity to Describe the Polarimetric State of a 3-D Field Within the Reverberating Chamber
}

\author{
Maurizio Migliaccio ${ }^{\circledR}$, Fellow, IEEE, Antonio Sorrentino ${ }^{\circledR}$, Member, IEEE, \\ Ferdinando Nunziata, Senior Member, IEEE, José Jorge Gil, and Sergio Cappa, Student Member, IEEE
}

\begin{abstract}
Reverberating chambers (RCs) are electrically large microwave enclosures in which a random electromagnetic 3-D, i.e., nonplanar, field is generated. To characterize such 3-D fields, a proper polarimetric decomposition is requested. In this article, a new set of parameters, namely the components of purity (CP), first proposed to the electromagnetic compatibility community, is presented. The CP parameters, which stem for the so-called trivial decomposition, are able to classify any 3-D polarimetric field into two states: regular and nonregular. Such characterization is of practical relevance, e.g., for $\mathrm{RC}$ isotropy testing.
\end{abstract}

Index Terms-Polarimetry, reverberating chamber (RC).

\section{INTRODUCTION}

$\mathbf{R}$ EVERBERATING chambers (RC) are reliable and costefficient facilities to perform electromagnetic compatibility (EMC) tests [1] since they emulate effective and reliable complex electromagnetic environments [2], [3]. RCs are used for a wide range of applications, e.g., testing antenna performance [4], [5], shielding effectiveness [6], [7], radiated emission test [8], [9], emulating wireless propagation channels [10]-[14], and biomedical measurements [15].

The RC is an electrically large metallic enclosure wherein the electromagnetic field is randomized using a stirring process [16], [17] that generates a random 3-D, i.e., nonplanar, electromagnetic field. To fully characterize its polarimetric properties, specialized descriptors must be used [18]-[23]. In fact, the nonplanar nature of the electromagnetic field calls for an appropriate 3-D decomposition [24]-[28], which leads to physically consistent parameters.

Manuscript received May 21, 2019; revised October 25, 2019, March 5, 2020 , and April 10,2020; accepted April 13, 2020. (Corresponding author: Maurizio Migliaccio.)

Maurizio Migliaccio, Antonio Sorrentino, Ferdinando Nunziata, and Sergio Cappa are with the Dipartimento di Ingegneria, Università degli Studi di Napoli Parthenope, 80133 Naples, Italy (e-mail: maurizio.migliaccio@uniparthenope. it; antonio.sorrentino@uniparthenope.it; ferdinando.nunziata@uniparthenope.it; sergio.cappa@uniparthenope.it).

José Jorge Gil is with the Departamento de Fisica Aplicada, Universidad de Zaragoza, 50009 Zaragoza, Spain (e-mail: ppgil@unizar.es).

Color versions of one or more of the figures in this article are available online at https://ieeexplore.ieee.org.

Digital Object Identifier 10.1109/TEMC.2020.2993898
Any 3-D decomposition starts with the estimate of the 3-D coherency matrix (R) [29], [30]. Then, compact parameters that carry on information about the state of the electromagnetic field are defined. Among them, the 3-D degree of polarimetric purity $P_{3 \mathrm{D}}$ [24] (bounded between 0 and 1) can be defined to measure the closeness of the polarization state to a fully polarized one (also known as pure state) [31]. Recently, the so-called index of polarimetric purity (IPP) decomposition was introduced in optics to decompose $P_{3 \mathrm{D}}$ according to parameters stemming for the trivial decomposition [24]. The IPP parameters, which were first presented and tested for RC electromagnetic field characterization in [29], decouple the information carried out $P_{3 \mathrm{D}}$ into two parts: first, the stability of the polarization ellipse $\left(P_{1}\right)$ that preserves its physical meaning of 2-D degree of polarization and second, the stability of propagation direction $\left(P_{2}\right)$ that is inherently related to 3-D states [29]. However, the IPP parameters are not able to represent important 3-D electromagnetic behaviors [30]-[37].

With this respect, a new set of parameters are introduced in [30]-[37] and they are hereafter first presented to the EMC community and applied to a set of meaningful microwave measurements carried out in the RC of the Università degli Studi di Napoli Parthenope, Naples, Italy. The new set of parameters is known as component of purity (CP) and splits $P_{3 \mathrm{D}}$ into three parameters, which now allow to represent a particular class of polarization states, i.e., the nonregular, whose polarimetric behavior cannot be described by the IPP.

In this article, we first show that the nonregular 3-D polarization states may occur in the loaded RC and they can only be represented by means of the $\mathrm{CP}$ decomposition. Hence, they can be used to make more accurate field isotropy testing in the RC that, as suggested in [38], should be performed using 3-D polarimetric approaches instead of using the conventional integrated 3-D linear measurements [1]. In fact, the conventional IEC 61000-4-21 standard [1] procedure is considered to be not enough when dealing with loaded RC configurations [38]. Hence, the proposed CP approach represents an important step to trigger the development of more accurate methods to test isotropy within a loaded RC.

The rest of this article is organized as follows. Section II describes the theoretical background on the polarimetric representation of a 3-D field. In Section III, the measurement setup, the procedures for measuring the 3-D field, and the experimental 
results are shown and discussed. Finally Section IV concludes this article.

\section{THEORETICAL BACKGROUND}

In this section, the theoretical background that underpins the description of the polarimetric properties of a generic 3-D electromagnetic field is detailed.

The complete characterization of the polarization properties of an electromagnetic field is given by the $3 \times 3 \mathbf{R}$ matrix [30]

$$
\mathbf{R}=\left\langle\mathbf{E} \otimes \mathbf{E}^{\dagger}\right\rangle=\left(\begin{array}{ccc}
\left\langle E_{x} E_{x}^{*}\right\rangle & \left\langle E_{x} E_{y}^{*}\right\rangle & \left\langle E_{x} E_{z}^{*}\right\rangle \\
\left\langle E_{y} E_{x}^{*}\right\rangle & \left\langle E_{y} E_{y}^{*}\right\rangle & \left\langle E_{y} E_{z}^{*}\right\rangle \\
\left\langle E_{z} E_{x}^{*}\right\rangle & \left\langle E_{z} E_{y}^{*}\right\rangle & \left\langle E_{z} E_{z}^{*}\right\rangle
\end{array}\right)
$$

where $\mathbf{E}$ is the Jones vector associated to the 3-D field whose components in an orthogonal linear reference frame are $E_{x}$, $E_{y}$, and $E_{z} . \otimes$ stands for the Kronecker product, $\dagger$ and $*$ stand for complex conjugate transposed and complex conjugate, respectively. Note that $\langle\cdot\rangle$ represents the time averaging. Since, by construction, $\mathbf{R}$ is a complex Hermitian and semidefinite positive matrix, it can be diagonalized as

$$
\mathbf{R}=\mathbf{U} \operatorname{diag}\left(\lambda_{1}, \lambda_{2}, \lambda_{3}\right) \mathbf{U}^{\dagger}=\sum_{k=1}^{3} \lambda_{k}\left(\mathbf{u}_{k} \otimes \mathbf{u}_{k}^{\dagger}\right)
$$

where $\mathbf{U}$ is a unitary matrix whose columns are the eigenvectors, i.e., $\mathbf{u}_{k}$, of $\mathbf{R}$, whereas $\lambda_{k}(k=1,2,3)$ are the nonnegative ordered eigenvalues, i.e., $\lambda_{1} \geq \lambda_{2} \geq \lambda_{3} \geq 0$ and $\operatorname{diag}(\cdot)$ is a $3 \times 3$ diagonal matrix where the elements of the main diagonal are specified in the brackets. $\mathbf{R}$ has the structure of a covariance matrix and the polarization state is completely described by means of the eigenvalues. Their sum is such that

$$
\operatorname{tr}(\mathbf{R})=\lambda_{1}+\lambda_{2}+\lambda_{3}=I
$$

where $I$ stands for the intensity of the field. Unlike the 2-D case, where the degree of polarization can be physically interpreted as the ratio between the polarized part and the total field intensity; in the 3-D case, this physical rationale in general can no longer be applied. This is due to the fact that $\mathbf{R}$ cannot be generally decomposed into its fully polarized and unpolarized parts [24].

A key parameter to describe the polarimetric state of a generic electromagnetic wave is the 3-D degree of polarization [22] that, in terms of invariants of $\mathbf{R}$, is given by [30]

$$
P_{3 \mathrm{D}}=\sqrt{\frac{1}{2} \frac{3 \operatorname{tr}\left(\mathbf{R}^{2}\right)}{\operatorname{tr}(\mathbf{R})^{2}}-1}
$$

where $P_{3 \mathrm{D}}$ is strictly connected to the eigenvalues of $\mathbf{R}$ and it is bounded into the $[0,1]$ interval. In fact, a pure 3-D state is characterized by $P_{3 \mathrm{D}}=1$, whereas a fully unpolarized 3-D state is characterized by $P_{3 \mathrm{D}}=0$ and results from a $\mathbf{R}$ matrix with three nonzero and equal eigenvalues.

Although $P_{3 \mathrm{D}}$ gives an overall measure of the polarimetric purity of a given 3-D polarization state [36], it does not provide complete information on all invariant polarimetric characteristics associated to the corresponding polarization matrix $\mathbf{R}[30]$. According to the approach provided in [24] and [27], which grounds on mathematical properties of the eigenvalues of $\mathbf{R}$, the structure of the polarization state of a generic 3-D wave can be modeled as a superposition of certain mutually incoherent states. With these respects, it is useful to proceed along the so-called trivial (or characteristic) decomposition of $\mathbf{R}$ [35]

$$
\mathbf{R}=P_{1} \mathbf{R}_{p}+\left(P_{2}-P_{1}\right) \mathbf{R}_{m}+\left(1-P_{2}\right) \mathbf{R}_{u-3 \mathrm{D}}
$$

where

$$
\begin{array}{r}
\mathbf{R}_{p}=\mathbf{U d i a g}(1,0,0) \mathbf{U} \\
\mathbf{R}_{m}=\frac{1}{2} \mathbf{U} \operatorname{diag}(1,1,0) \mathbf{U}^{\dagger} \\
\mathbf{R}_{u-3 \mathrm{D}}=\frac{1}{3} \mathbf{I}
\end{array}
$$

with $\mathbf{I}$ being the $3 \times 3$ identity matrix. $P_{1}$ and $P_{2}$ are termed IPP and they weight the elementary states that characterize the generic 3-D state [30]

$$
P_{1}=\frac{\lambda_{1}-\lambda_{2}}{I} \quad \text { and } \quad P_{2}=\frac{\lambda_{1}+\lambda_{2}-2 \lambda_{3}}{I} .
$$

It is important to note that $P_{1}$ deals with the stability of the polarization ellipse, preserving its physical meaning of 2-D degree of polarization, whereas $P_{2}$ provides information on the stability of propagation direction [29]. The IPP parameters must satisfy the following inequality:

$$
0 \leq P_{1} \leq P_{2} \leq 1
$$

Also note that by using (3), (8), and (9), $P_{3 \mathrm{D}}$ can be expressed in terms of $P_{1}$ and $P_{2}$ according to the following quadratic average:

$$
P_{3 \mathrm{D}}=\sqrt{\frac{3}{4} P_{1}^{2}+\frac{1}{4} P_{2}^{2}} .
$$

It must be explicitly noted that a deeper physical understanding of the trivial decomposition and, therefore, a better interpretation of the physical features of a 3-D polarization state depend strongly on the matrix $\mathbf{R}_{m}$ associated with the so-called discriminating state.

It is important to remark that the trivial decomposition physically interpreted by means of the IPP parameters does not allow to represent any 3-D polarimetric state [35]. In order to better clarify this key point, meaningful showcases are illustrated.

The matrices $\mathbf{R}_{p}$ and $\mathbf{R}_{u-3 \mathrm{D}}$ always represent a pure 3-D state and a 3-D unpolarized state, respectively. With respect to $\mathbf{R}_{m}$, following different cases must be distinguished.

1) When $P_{1}=P_{2}, \mathbf{R}_{m}$ does not play any role in (4) that becomes

$$
\mathbf{R}=P_{1} \mathbf{R}_{p}+\left(1-P_{1}\right) \mathbf{R}_{u-3 \mathrm{D}} .
$$

In this particular case, the 3-D polarization state can be considered an incoherent composition of a pure state and an unpolarized 3-D state.

In general, $P_{1} \neq P_{2}$, so that the matrix $\mathbf{R}_{m}$ plays a key role in the trivial decomposition. Two important cases must be distinguished, which are as follows.

1) When $\mathbf{R}_{m}$ is a real matrix, necessarily $\mathbf{R}_{m}=\frac{1}{2} \mathbf{I}=$ $\mathbf{R}_{u-2 \mathrm{D}}$ so that $\mathbf{R}_{m}$ always corresponds to a 2-D unpolarized state (i.e., the electric field associated to this electromagnetic wave evolves fully randomly in a fixed 
plane). Hence, when $\mathbf{R}_{m}$ is a real matrix, according to (4), the structure of the polarization state can be fully described by means of the incoherent superposition of a pure state $\left(\mathbf{R}_{p}\right)$, a 3-D unpolarized state $\left(\mathbf{R}_{u-3 \mathrm{D}}\right)$, and a 2-D unpolarized state $\left(\mathbf{R}_{u-2 \mathrm{D}}\right)$. The generic states for which this physical rationale applies are termed regular states [35].

2) When $\mathbf{R}_{m}$ is not a real matrix, there is no orthogonal (also known as laboratory) reference frame that diagonalizes the rank-3 $\mathbf{R}_{m}$ matrix and a unitary rotation matrix (6) must be used. The polarization states such that $\mathbf{R}_{m}$ is not a real matrix are termed nonregular states [35].

The concept of nonregularity relies on certain properties of the coherency matrix relative to its transformation under arbitrary rotations provided by the orthogonal similarity transformation $\mathbf{Q}_{0}$. Hence, it is instructive to split $\mathbf{R}_{m}$ into its real $\left(\mathbf{R}_{R}\right)$ and imaginary $\left(\mathbf{R}_{I}\right)$ parts. As a result, we get to the intrinsic coherency matrix $\mathbf{R}_{0}$ that contains the same information of $\mathbf{R}_{m}$ and is given by [30]

$$
\begin{gathered}
\mathbf{R}_{0} \equiv \mathbf{Q}_{0} \mathbf{R} \mathbf{Q}_{0}^{\mathrm{T}}=\mathbf{Q}_{0} \mathbf{R}_{R} \mathbf{Q}_{0}^{\mathrm{T}}+i \mathbf{Q}_{0} \mathbf{R}_{I} \mathbf{Q}_{0}^{\mathrm{T}} \\
=\left(\begin{array}{ccc}
a_{1} & 0 & 0 \\
0 & a_{2} & 0 \\
0 & 0 & a_{3}
\end{array}\right)+\left(\begin{array}{ccc}
0 & -i n_{03} & i n_{02} \\
i n_{03} & 0 & -i n_{01} \\
-i n_{02} & i n_{01} & 0
\end{array}\right)
\end{gathered}
$$

where $\mathrm{T}$ stands for transpose, $i$ is the imaginary unit, and the nonnegative real parameters $a_{j s^{\prime}}$ are the eigenvalues of $\mathbf{R}_{R}$, also known as principal intensities. $\mathbf{Q}_{0}$ is defined so as to satisfy $a_{1} \geq a_{2} \geq a_{3}$, and $n_{0 j s^{\prime}}$ are the components of the intrinsic angular momentum vector $\mathbf{n}_{0} \equiv\left(n_{01}, n_{02}, n_{03}\right)^{\mathrm{T}}$ of the state with respect to the so-called intrinsic reference frame $X_{0} Y_{0} Z_{0}$ [35]. CP parameters, namely the degree of linear polarization $P_{l}$, the degree of circular polarization $P_{c}$, and the degree of directionality $P_{d}$ are defined from $\mathbf{R}_{0}$ as follows [30], [34]:

$$
\begin{aligned}
P_{l} & =\frac{a_{1}-a_{2}}{a_{1}+a_{2}+a_{3}} \\
P_{c} & =\frac{\sqrt{n_{01}^{2}+n_{02}^{2}+n_{03}^{2}}}{a_{1}+a_{2}+a_{3}} \\
P_{d} & =\frac{a_{1}+a_{2}-2 a_{3}}{a_{1}+a_{2}+a_{3}} .
\end{aligned}
$$

The three $\mathrm{CP}$ parameters are invariant under orthogonal transformations (rotations in the real space) and provide a meaningful framework to describe any 3-D polarization state in terms of quantities that are intrinsic for each given state [34], [35]. Other mathematical properties associated with CP parameters as well as their expressions in terms of the nine 3-D Stokes parameters are provided in [20]-[28]. It is often useful to combine $P_{l}$ and $P_{c}$ into an overall degree of elliptical purity $P_{e}$ that accounts jointly the degrees of linear and circular polarization [36]

$$
P_{e}=\sqrt{P_{l}^{2}+P_{c}^{2}} \text {. }
$$

The parameter $P_{d}$ measures how close the polarization state described by $\mathbf{R}$ is to a 2-D polarization state. It is also worth to recall that $P_{3 \mathrm{D}}$ can be expressed in terms of CP parameters as

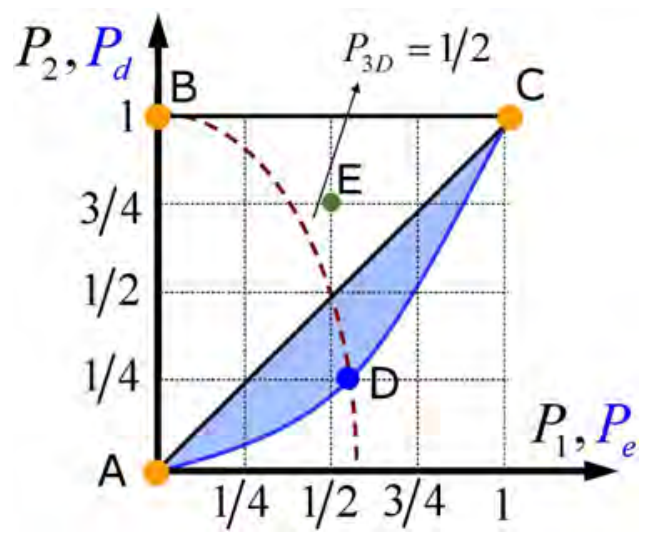

Fig. 1. Feasible region for IPP parameters $\left(P_{1}, P_{2}\right)$ is determined by the triangle "ABC," whereas the feasible region for CP parameters $\left(P_{e}, P_{d}\right)$ also includes the shadowed blue area. For both representations, the points " $A$ " and "C" correspond to regular states. The shadowed blue area (except for the straight line $\overline{\mathrm{AC}}$ ) is exclusive of nonregular states, whereas the points inside the triangle "ABC" can result from either regular or nonregular states. The point "E" corresponds to the nonregular state measured in the proposed loaded RC configuration.

follows:

$$
P_{3 \mathrm{D}}=\sqrt{\frac{3}{4}\left(P_{l}^{2}+P_{c}^{2}\right)+\frac{1}{4} P_{d}^{2}}=\sqrt{\frac{3}{4} P_{e}^{2}+\frac{1}{4} P_{d}^{2}} .
$$

The CP parameters always satisfy the following inequalities:

$$
\begin{gathered}
P_{l}^{2} \leq P_{d}^{2} \leq 1 \\
0 \leq P_{e}^{2} \leq 1 .
\end{gathered}
$$

According to the aforementioned rationale, $\mathbf{R}$ represents a regular state if and only if $P_{e}=P_{1}$ and $P_{d}=P_{2}$. Therefore, the theoretical framework that includes the trivial decomposition and the CP parameters allows a physically consistent interpretation of 3-D states distinguishing between regular and nonregular states.

The peculiar features and differences between IPP and CP indicators can be simply understood by contrasting IPP $\left(P_{1}, P_{2}\right)$ and $\mathrm{CP}\left(P_{e}, P_{d}\right)$ parameters on the 2-D purity space depicted in Fig. 1. The vertical (horizontal) axis includes $P_{2}$ and $P_{d}\left(P_{1}\right.$ and $P_{e}$ ). The feasible region related to the IPP parameters consists of the triangle "ABC," whereas the feasible region of the $\mathrm{CP}$ parameters consists of the "ABC" triangle augmented by the shadowed blue area.

The points "A" $\left(P_{3 \mathrm{D}}=0\right)$ and "C" $\left(P_{3 \mathrm{D}}=1\right)$ represent regular fully unpolarized and polarized 3-D states, respectively. The straight line $\overline{\mathrm{AC}}$ is exclusive of regular states since it calls for $P_{1}=P_{2}$. The shadowed blue area (except for the $\overline{\mathrm{AC}}$ segment) is exclusive of nonregular states since it calls for $P_{2} \neq P_{d}$ and $P_{1} \neq P_{e}$. The triangle "ABC" (except for the points "A," "C," and the segment $\overline{\mathrm{AC}}$ ) calls for either regular or nonregular states. In particular, for a given $P_{3 \mathrm{D}}$ value, IPP and CP parameters describe a common elliptical branch and they coincide only and only if a regular state is in place.

As an illustrative example, the elliptical branch that corresponds to $P_{3 \mathrm{D}}=0.5$ is depicted as dashed red line in Fig. 1. One 


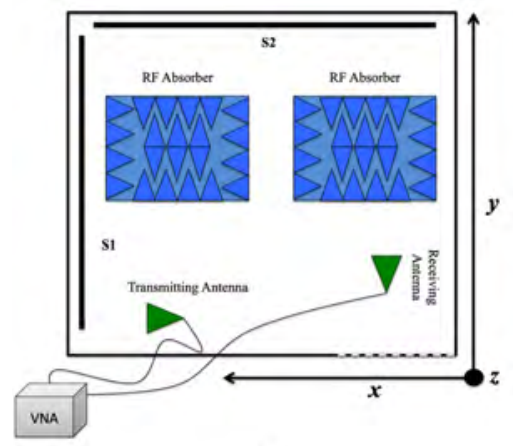

(a)

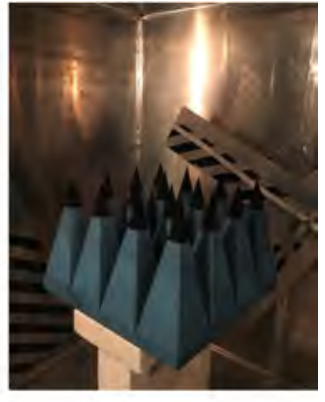

(b)
Fig. 2. RC of the UNP. (a) RC configuration, (b) RF standard absorber.

can note that it includes all the discriminating states [i.e., $\mathbf{R}_{m}$ matrix in (5)] that result from $P_{1}=0$ and $P_{2}=1$. Regular states are achieved if and only if $P_{1}=P_{e}$ and $P_{2}=P_{d}$. It is worth showing the point " $D$ " that, belonging to the shadowed region, results from a nonregular state. In particular, as shown in [37], the point " $D$ " represents a state with maximum nonregularity.

To summarize, the framework that lies on the trivial decomposition together with the $\mathrm{CP}$ allows interpreting and classifying polarization states in an objective way and provides a proper way to distinguish between regular and nonregular states.

\section{EXPERIMENTAL RESULTS}

In this section, a meaningful set of experiments are presented and discussed to show the physical significance and the interconnection among $P_{3 \mathrm{D}}$, IPP and CP parameters, and the limits of IPP.

Measurements are performed using a $8-\mathrm{m}^{3}$ cubic RC whose setup is depicted in Fig. 2. Within the chamber, three mechanical stirrers, S1, S2, and S3, operate in continuous mode to randomize the input electromagnetic field. S1 and $\mathrm{S} 2$ are placed on the left wall and in front of the entrance door, respectively, whereas S3 is placed on the top of the chamber. Two Narda Waveguide Horn Antennas certified to work in $X$-band, i.e., from 8.5 to $12.4 \mathrm{GHz}$ frequency range, are used. The two antennas do not face each other, i.e., a non-line-of-sight configuration is adopted, and they are placed at about $1 \mathrm{~m}$ on the floor on Styrofoam supports that are transparent to the electromagnetic field. A twoport Agilent Technologies vector network analyzer is used to measure the complex parameter $\mathrm{S}_{21}$. It is important to note that the statistics of $S_{21}$ are equivalent to the statistics of the Cartesian field components inside an RC [39], [40].

The calibration procedure is carried out using the CalKit 85400 and the response and isolation procedure. All the experiments refer to a $12-\mathrm{GHz}$ electromagnetic field and different $\mathrm{RC}$ configurations are considered, which are obtained by varying the number and the position of the microwave absorbers. The measurements are performed by aligning the transmitting antenna to each of the three axis of an orthogonal Cartesian reference system centered into the transmitting antenna. For each transmitted linearly polarized wave, the receiving antenna is aligned to each of the three axis of an orthogonal Cartesian

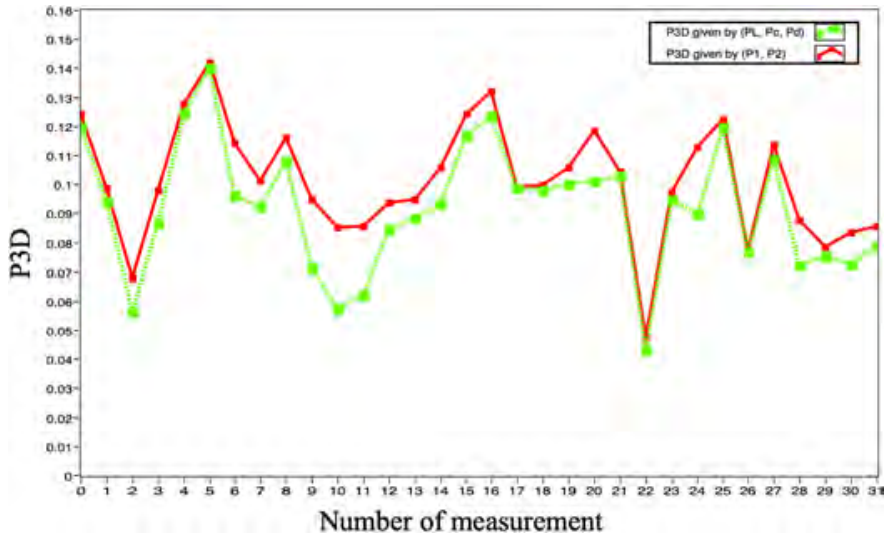

Fig. 3. Unloaded RC. $P_{3 \mathrm{D}}$ evaluated measuring CPs (green dotted line) and IPPs (red dot continuous line). The transmitting antenna is polarized along the $\mathrm{X}$-axis.

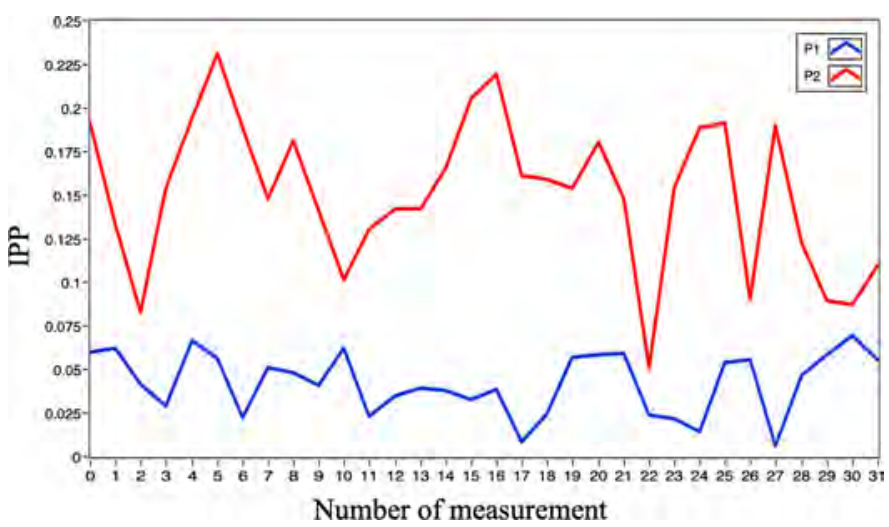

Fig. 4. Unloaded RC. $P_{1}$ (blue line) and $P_{2}$ (red line) are shown when the transmitting antenna is polarized along the $\mathrm{x}$-axis.

reference system centered into the receiving antenna [29]. For each receiving antenna position, 32 independent measurements are obtained by averaging 500 field samples. It is worth noting that since the continuous stirring mechanism is adopted, the geometry of the RC and its boundary conditions are continuously changing during the measurement time. Hence, rotating the antenna is not expected to significantly affect the measurement process [17]. In addition, the average field measured within a well-stirred RC has been shown in [41] to be independent of the antennas used. The IPP and CP parameters are then offline evaluated and analyzed using the LabVIEW environment.

The first experiment refers to the unloaded RC configuration and aims at verifying that IPP (11) and CP (16) parameters allow the same physical interpretation of the field within the $\mathrm{RC}$ when a regular state is in place. In Fig. 3, the transmitting antenna is aligned with the $\mathrm{x}$-axis of a Cartesian reference system and $P_{3 \mathrm{D}}$ is shown in red continuous line (IPP) and green dotted line (CP). As expected, the two lines are almost completely overlapped resulting in $P_{3 \mathrm{D}}$ values (mean \pm standard deviation) equal to $0.079 \pm 0.038$ and $0.076 \pm 0.038$ for the IPP and CP cases, respectively (see Table I). $P_{3 \mathrm{D}}$ values obtained in this experiment, according to what formerly experienced in [23] 
TABLE I

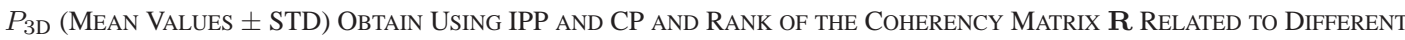
RC Configuration and Measured When the Transmitting ANTENna Is Aligned With the X, Y, AND Z AXES

\begin{tabular}{ccccc}
\hline $\begin{array}{c}\text { Transmitted } \\
\text { Polarization }\end{array}$ & $\begin{array}{c}\text { RC } \\
\text { Configuration }\end{array}$ & $\begin{array}{c}\left\langle P_{3 \mathrm{D}}\right\rangle \pm \sigma_{P_{3 D}} \\
\text { in terms of } \\
\left(P_{e}, P_{d}\right)\end{array}$ & $\begin{array}{c}\left\langle P_{3 \mathrm{D}}\right\rangle \pm \sigma_{P_{3 D}} \\
\text { in terms of } \\
\left(P_{1}, P_{2}\right)\end{array}$ & $\begin{array}{c}\text { Rank of } \\
\mathbf{R}\end{array}$ \\
\hline $\boldsymbol{x}$ & No Abs & $0.076 \pm 0.038$ & $0.079 \pm 0.038$ & 3 \\
& $1 \mathrm{Abs}$ & $0.812 \pm 0.030$ & $0.714 \pm 0.029$ & 2 \\
& $2 \mathrm{Abs}$ & $0.850 \pm 0.035$ & $0.854 \pm 0.034$ & 2 \\
\hline $\boldsymbol{y}$ & No Abs & $0.134 \pm 0.052$ & $0.142 \pm 0.056$ & 3 \\
& $1 \mathrm{Abs}$ & $0.820 \pm 0.045$ & $0.823 \pm 0.046$ & 2 \\
$z$ & $2 \mathrm{Abs}$ & $0.826 \pm 0.048$ & $0.829 \pm 0.051$ & 2 \\
\hline \multirow{z}{*}{ No Abs } & $0.137 \pm 0.035$ & $0.138 \pm 0.038$ & 3 \\
& $1 \mathrm{Abs}$ & $0.580 \pm 0.078$ & $0.432 \pm 0.076$ & 3 \\
& $2 \mathrm{Abs}$ & $0.609 \pm 0.099$ & $0.606 \pm 0.099$ & 2 \\
\hline
\end{tabular}

TABLE II

CP AND IPP VALUES (MEAN VAlues \pm STD) RELATED TO DifFERENT RC CONFIGURATION AND MEASUREd When the Transmitting ANTENNA Is ALIGNED With THE X, Y, AND Z AXES

\begin{tabular}{|c|c|c|c|c|c|c|c|}
\hline $\begin{array}{l}\text { Transmitted } \\
\text { Polarization }\end{array}$ & $\begin{array}{c}\text { RC } \\
\text { Configuration }\end{array}$ & $\left\langle P_{l}\right\rangle \pm \sigma_{P_{l}}$ & $\left\langle P_{c}\right\rangle \pm \sigma_{P_{c}}$ & $\left\langle P_{d}\right\rangle \pm \sigma_{P_{d}}$ & $\left\langle P_{e}\right\rangle \pm \sigma_{P_{e}}$ & $\left\langle P_{1}\right\rangle \pm \sigma_{P_{1}}$ & $\left\langle P_{2}\right\rangle \pm \sigma_{P_{2}}$ \\
\hline \multirow[t]{3}{*}{ 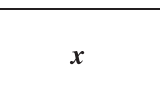 } & No Abs & $0.079 \pm 0.009$ & $0.010 \pm 0.007$ & $0.135 \pm 0.008$ & $0.080 \pm 0.006$ & $0.055 \pm 0.006$ & $0.125 \pm 0.008$ \\
\hline & $1 \mathrm{Abs}$ & $0.807 \pm 0.040$ & $0.062 \pm 0.008$ & $0.962 \pm 0.006$ & $0.654 \pm 0.017$ & $0.646 \pm 0.055$ & $0.886 \pm 0.072$ \\
\hline & $2 \mathrm{Abs}$ & $0.872 \pm 0.046$ & $0.066 \pm 0.052$ & $0.975 \pm 0.010$ & $0.876 \pm 0.059$ & $0.889 \pm 0.045$ & $0.989 \pm 0.072$ \\
\hline \multirow{3}{*}{$y$} & No Abs & $0.100 \pm 0.062$ & $0.085 \pm 0.038$ & $0.086 \pm 0.066$ & $0.139 \pm 0.067$ & $0.069 \pm 0.034$ & $0.260 \pm 0.121$ \\
\hline & $1 \mathrm{Abs}$ & $0.782 \pm 0.060$ & $0.118 \pm 0.056$ & $0.895 \pm 0.024$ & $0.803 \pm 0.095$ & $0.848 \pm 0.055$ & $0.892 \pm 0.104$ \\
\hline & $2 \mathrm{Abs}$ & $0.795 \pm 0.061$ & $0.167 \pm 0.062$ & $0.856 \pm 0.049$ & $0.815 \pm 0.111$ & $0.831 \pm 0.054$ & $0.972 \pm 0.094$ \\
\hline \multirow{3}{*}{$z$} & No Abs & $0.068 \pm 0.042$ & $0.083 \pm 0.032$ & $0.167 \pm 0.095$ & $0.144 \pm 0.042$ & $0.070 \pm 0.033$ & $0.231 \pm 0.075$ \\
\hline & $1 \mathrm{Abs}$ & $0.353 \pm 0.123$ & $0.330 \pm 0.077$ & $0.750 \pm 0.058$ & $0.506 \pm 0.095$ & $0.151 \pm 0.065$ & $0.805 \pm 0.079$ \\
\hline & $2 \mathrm{Abs}$ & $0.476 \pm 0.157$ & $0.155 \pm 0.077$ & $0.892 \pm 0.056$ & $0.521 \pm 0.050$ & $0.551 \pm 0.059$ & $0.953 \pm 0.075$ \\
\hline
\end{tabular}

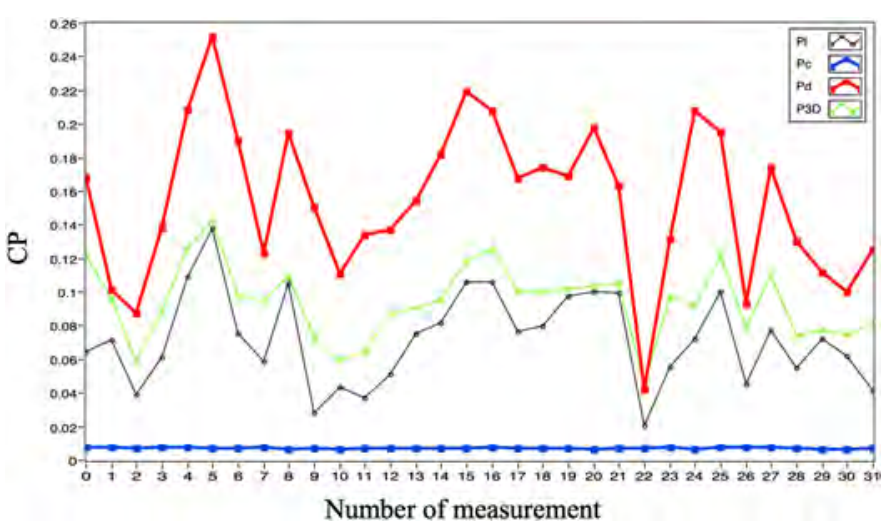

Fig. 5. Unloaded RC. $P_{l}$ (dotted black line), $P_{c}$ (blue line), $P_{d}$ (red line), and $P_{3 \mathrm{D}}$ (green dotted line) are shown when the transmitting antenna is polarized along the $\mathrm{x}$-axis.

through independent measurements, are compatible with a fully unpolarized 3-D state. This is further confirmed by the analysis of IPP (see Fig. 4), which shows that as expected, $P_{1}<P_{2}$ and both the parameters result in very low values. In particular, $P_{1}$ and $P_{2}$ values (mean \pm standard deviations) are $0.055 \pm 0.006$ and $0.125 \pm 0.008$, respectively (see Table II). Hence, a 3-D unpolarized state is in place. The behavior of $\mathrm{CP}$ parameters is depicted in Fig. 5 where one can see that they result, as expected, in very low values. In addition, by using $P_{e}$ (12), one can note that $P_{e} \cong P_{l} \cong P_{1}$, i.e., $P_{c} \cong 0$, whereas $P_{d} \cong P_{2}$. Hence, the rank-3 $\mathbf{R}$ matrix represents a 3-D regular state [35]. The mean values \pm standard deviations of both IPPs and CPs values are listed in Table II. Similar results are obtained when the transmitting antenna is polarized along the $\mathrm{y}$ and $\mathrm{z}$ axes (see Tables I and II).

Next experiments refer to the RC loaded with absorbers and aim at providing, on one side, an understanding of the difference between IPP and CP parameters; on the other side, they demonstrate that the RC is a very versatile environment that allows generating any polarimetric state, including the nonregular one. Hence, an Emerson and Cuming RF standard absorber block, consisting of 16 conical pieces arranged as a $4 \times 4$ array, is used [see Fig. 2(b)] to load the RC. The absorber block is placed in the RC corner, in front of the transmitting antenna and about $1 \mathrm{~m}$ above the floor [see Fig. 2(a)]. The taper length is about $20 \mathrm{~cm}$.

The second experiment refers to the transmitting antenna polarized along the $\mathrm{x}$-axis. $P_{3 \mathrm{D}}$ values evaluated using the IPP and $\mathrm{CP}$ parameters are equal to $0.714 \pm 0.030$ and $0.812 \pm 0.029$, respectively (see Table I). The degree of purity increased significantly with respect to the unloaded case witnessing that the absorbers reduce the randomness of the field within the RC. The behavior of $P_{1}$ and $P_{2}$ is depicted in Fig. 6. $P_{1}$ and $P_{2}$ values $(0.646 \pm 0.055$ and $0.886 \pm 0.072$, respectively) increased with respect to the unloaded case (see Table II). In particular, $P_{2}$ is closer to 1 , witnessing that the direction of propagation is practically fixed and well defined. Moreover, $\mathbf{R}$ is now a rank-2 matrix, witnessing that a 2-D polarization state is in place. Such result is confirmed by CP parameters (see Fig. 7), where $P_{l}$ (black curve), $P_{c}$ (blue curve), and $P_{d}$ (red curve) are shown. Even in this case, $P_{l}$ and $P_{d}$ increased with respect to the unloaded case, whereas $P_{c}$ is still negligible. The mean value and the standard 


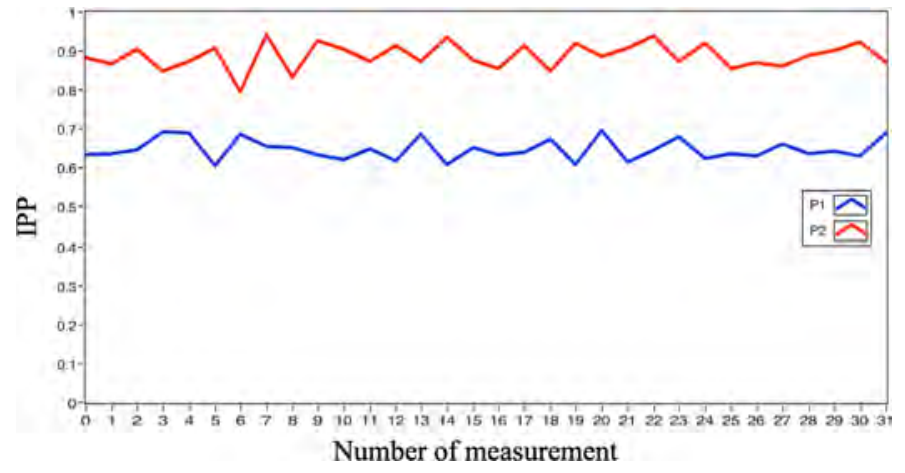

Fig. 6. RC loaded with one piece of absorber. $P_{1}$ (blue line) and $P_{2}$ (red line) are shown when the transmitting antenna is polarized along the $\mathrm{x}$-axis.

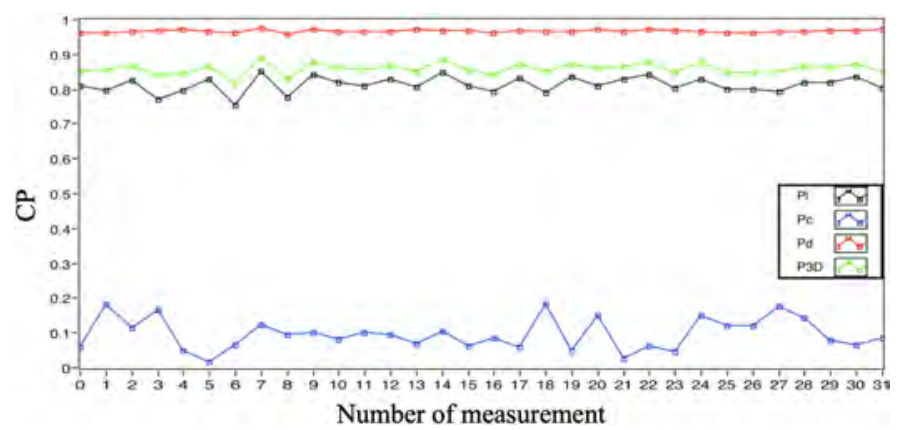

Fig. 7. $\quad$ RC loaded with one piece of absorber. $P_{l}$ (dotted black line), $P_{c}$ (blue line), $P_{d}$ (red line), and $P_{3 \mathrm{D}}$ (green dotted line) are shown when the transmitting antenna is polarized along the $\mathrm{x}$-axis.

deviation of IPP and CP parameters are listed in Table II. In summary, one can note that $P_{d} \cong P_{2} \cong 1,0 \leq P_{e} \cong P_{1}<1$, and $1 / 2 \leq P_{3 \mathrm{D}}<1$. The field received within the well-stirred $\mathrm{RC}$ loaded with one block of absorbers is a 2-D regular polarization state and both IPP and CP parameters still provide the same information. Similar results are obtained when the transmitting antenna is aligned with the $y$-axis (see Tables I and II).

The third experiment refers to the transmitting antenna polarized along the z-axis. $P_{3 \mathrm{D}}$ values evaluated using the IPP and $\mathrm{CP}$ parameters are equal to $0.432 \pm 0.076$ and $0.580 \pm 0.078$, respectively (see Table I). It can be noted that $P_{3 \mathrm{D}}$ decreased with respect to the previous cases witnessing that a more random configuration is achieved when the transmitting antenna is $z$-polarized. The behavior of $P_{1}$ and $P_{2}$ is depicted in Fig. 8 (see blue and red continuous lines, respectively). It is worth pointing out that in this case, the IPP parameters do not offer additional information on the polarimetric properties of this electromagnetic field. In fact, as usual, $0 \leq P_{1} \leq P_{2} \leq 1$; hence, since $1 / 2 \leq P_{3 \mathrm{D}} \leq 1$, we can only say that a partially polarized wave is in place. However, the polarimetric state can be either 2-D or 3-D.

To further shed light on this ambiguous case, CP parameters are used (see Fig. 9). The mean values of the CP parameters are listed in Table II, which show that $0 \leq P_{d}<P_{2} \cong 1$ and $0 \leq P_{1}<P_{e}<1$; in addition, a rank-3 of $\mathbf{R}$ matrix is obtained.

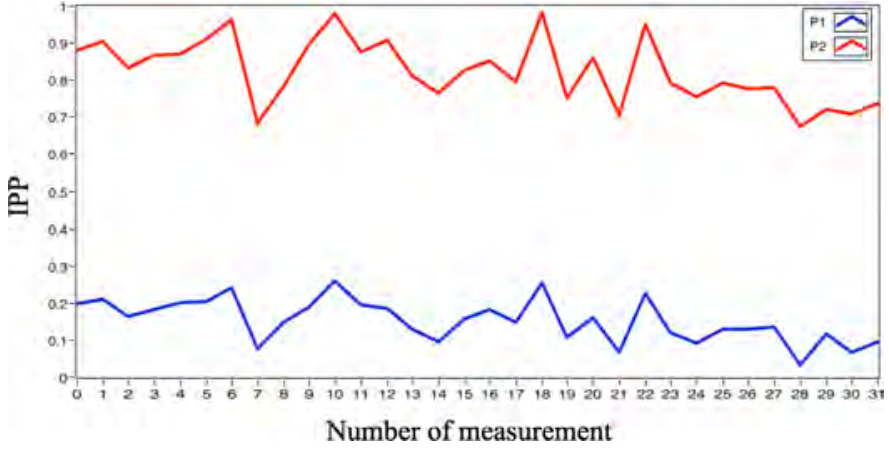

Fig. 8. RC loaded with one piece of absorber. $P_{1}$ (blue line) and $P_{2}$ (red line) are shown when the transmitting antenna is polarized along the $\mathrm{z}$-axis.

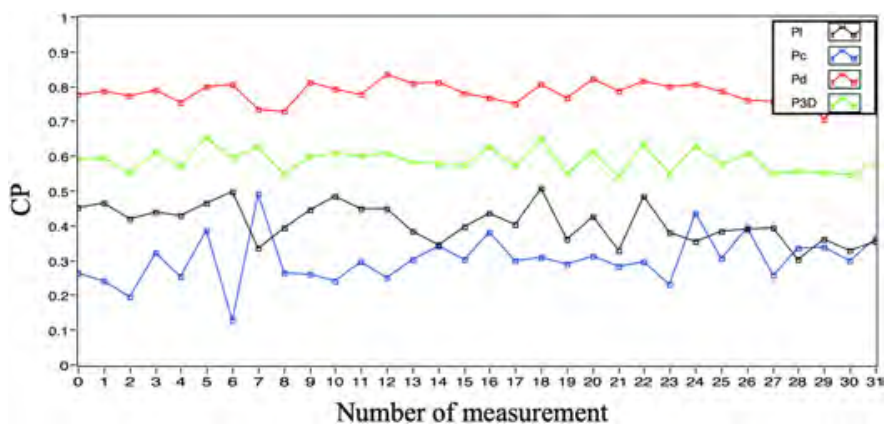

Fig. 9. RC loaded with one piece of absorber. $P_{l}$ (dotted black line), $P_{c}$ (blue line), $P_{d}$ (red line), and $P_{3 \mathrm{D}}$ (green dotted line) are shown when the transmitting antenna is polarized along the $\mathrm{z}$-axis.

Hence, the CP parameters witness that a 3-D nonregular polarization state is achieved.

From a physical view point, the generation of this nonregular state is likely due to the fact that the loaded RC is no longer an isotropic environment. In detail, when the antenna is moved in the azimuth plane (e.g., when the antenna is aligned along the $\mathrm{x}$ and $\mathrm{y}$ axes), the $\mathrm{RC}$ configuration is still isotropic since both the antenna opening and the RF absorber are at the same height. When the antenna points along the $z$-direction, a different scenario is in place since the antenna opening is at a different height with respect to the RF absorber block and it faces to the RC metallic floor. This scenario breaks the RC symmetry resulting in a nonisotropic environment, which allows obtaining 3-D electromagnetic fields whose polarimetric state belongs to the nonregular class.

The last set of measurements is made loading the RC with two RF absorbers. In particular, an additional absorber (of the same type of the previous one) is placed in the RC corner, in front of the receiving antenna and about $1 \mathrm{~m}$ above the floor [see Fig. 2(a)]. In this case, CP and IPP mean values confirm the results achieved when only one RF absorber is used, i.e., a partially polarized wave is in place.

When the transmitting antenna is aligned with the $\mathrm{x}$-axis the mean $P_{3 \mathrm{D}}$ values obtained using IPP and CP are equal to $0.850 \pm 0.035$ and $0.854 \pm 0.034$, respectively. The mean value and the standard deviation of IPP and CP parameters are listed in 
Table II. In summary, one can note that $P_{d} \cong P_{2} \cong 1,0 \leq P_{e} \cong$ $P_{1}<1$, and $1 / 2 \leq P_{3 \mathrm{D}}<1$. Hence, the polarimetric structure is now a 2-D one [35]. Similar results can be obtained when the transmitting antenna is aligned with the y-axis (see Tables I and II). It is interesting to note that when the antenna is aligned with the z-axis, the mean $P_{d}$ value is equal to $0.892 \pm 0.056$. Hence, $P_{d} \cong P_{2} \cong 1$ and $P_{e} \cong P_{1} \cong 1$ (see Table II). Moreover, $P_{e} \cong P_{1}$ (see Table II). Hence, unlike the previous experiment, in this case, a regular state is obtained and both CP and IPP parameters provide the same information. This result shows that the number and the position of the absorbers significantly affect the polarimetric behavior of the field inside the RC.

\section{CONCLUSION}

In this article, a new set of 3-D polarimetric $\mathrm{CP}$ parameters was first presented to the EMC community. The CP decomposition was first experimentally tested in this article where it is proven to be of physical interest. The main outcomes can be summarized as follows.

1) The CP decomposition provided a step forward with respect to the IPP decomposition in distinguishing between regular and nonregular states, overcoming the IPP decomposition limits.

2) The RC allowed emulating both regular and nonregular states. Nonregular states were associated to loaded RC.

3) Nonregular states occurring in the loaded RC were likely connected to the isotropy loss. Hence, the CP decomposition could be used to support more accurate field isotropy testing in the RC.

\section{REFERENCES}

[1] Reverberation Chamber Test Method, IEC Standard 61 000-4-21, International Electronical Commission, Geneva, Switzerland, 2011.

[2] "TP for 37.976: LTE MIMO OTA test plan for reverberation chamber based methodologies, azimuth systems, bluetest, CTTC," 3rd Generation Partnership Project, Sophia Antipolis, France, Tech. Rep. RAN4 - R4111690, 2011.

[3] A. Sorrentino, F. Nunziata, G. Ferrara, and M. Migliaccio, "Reverberating chamber profile identification," IET Microw. Antennas Propag., vol. 6, no. 13 , pp. $1468-1472,2012$.

[4] M. Á. García-Fernndez, D. Carsenat, and C. Decroze, "Antenna gain and radiation pattern measurements in reverberating chamber using Doppler effect," IEEE Trans. Antennas Propag., vol. 62, no. 10, pp. 5389-5394, Oct. 2014.

[5] A. Hussain, A. A. Glazunov, B. Einarsson, and P-S. Kildal, "Antenna measurements in reverberation chamber using USRP," IEEE Trans. Antennas Propag., vol. 64, no. 3, pp. 1152-1157, Mar. 2016.

[6] A. Gifuni et al., "On the evaluation of the shielding effectiveness of an electrically large enclosure," Adv. Electromagn., vol. 1, no. 5, pp. 84-91, 2012.

[7] C. L. Holloway et al., "Use of reverberation chambers to determine the shielding effectiveness of physically small, electrically large enclosures and cavities," IEEE Trans. Electromagn. Compat., vol. 50, no. 4, pp. 770782, Nov. 2008.

[8] M. Alparone et al., "Hydrogen-based hybrid power unit for light vehicles: Assessment of energy performance and radiated electromagnetic emissions," IL Nuovo Cimento C, vol. 41, no. 6, 2018, Art. no. 225.

[9] K. A. Remley, C. J. Wang, D. F. Williams, J. J. aan den Toorn, and C. L. Holloway, "A significance test for reverberation-chamber measurement uncertainty in total radiated power of wireless devices," IEEE Trans. Electromagn. Compat., vol. 58, no. 1, pp. 207-219, Feb. 2008.

[10] A. Sorrentino, G. Ferrara, and M. Migliaccio, "Kurtosis index to characterize near LOS conditions in reverberating chambers," IET Microw. Antennas Propag., vol. 7, no. 3, pp. 175-179, 2013.
[11] C. L. Holloway, H. A. Shah, R. J. Pirk, K. A. Remley, D. A. Hill, and J. Ladbury, "Early time behavior in reverberating chambers and its effect on the relationship between coherence bandwidth, chamber decay time, RMS delay spread, and the chamber buildup time," IEEE Trans. Electromagn. Compat., vol. 54, no. 4, pp. 714-725, Aug. 2012.

[12] A. Sorrentino, A. Gifuni, G. Ferrara, and M. Migliaccio, "Mode-stirred reverberating chamber autocorrelation function: Model, multifrequency measurements and applications," IET Sci. Meas. Technol., vol. 9, no. 5, pp. 547-554, 2015.

[13] A. Sorrentino, G. Ferrara, and S. Cappa, "Experimental Rice factor distribution within a reverberating chamber," IEEE Antennas Wireless Propag. Lett., vol. 16, pp. 2911-2914, 2017.

[14] A. Sorrentino, A. Gifuni, G. Ferrara, and M. Migliaccio, "Mode-stirred reverberating chamber Doppler spectra: Multi-frequency measurements and empirical model," IET Microw. Antennas Propag., vol. 8, no. 15, pp. 1356-1362, 2014.

[15] Y. El-Saboni, M. K. Magill, G. A. Conway, S. L. Cotton, and W. G. Scanlon, "Measurement of deep tissue implanted antenna efficiency using a reverberation chamber," IEEE J. Electromagn., RF, Microw. Med. Biol., vol. 1, no. 2, pp. 90-97, Dec. 2017.

[16] R. Serra et al., "Reverberation chambers à la carte: An overview of different mode-stirring techniques," IEEE Electromagn. Compat. Mag., vol. 6, no. 1, pp. 63-78, Jan.-Mar. 2017.

[17] D. A. Hill, Electromagnetic Fields in Cavities: Deterministic and Statistical Theories. New York, NY, USA: IEEE Press, 2009.

[18] L. R. Arnaut, "Compound exponential distributions for undermoded reverberation chambers," IEEE Trans. Electromagn. Compat., vol. 44, no. 3 , pp. 442-457, Aug. 2002.

[19] L. R. Arnaut, "Copulas, outliers, and rogue states of nonelliptic fields and energy in electromagnetic reverberation," IEEE Trans. Electromagn. Compat., vol. 58, no. 2, pp. 371-384, Apr. 2016.

[20] P. Roman, "Generalized Stokes parameters for waves with arbitrary form," IL Nuovo Cimento, vol. 13, no. 5, pp. 974-982, 1959.

[21] E. Wolf, Introduction to the Theory of Coherence and Polarization of Light. Cambridge, U.K.: Cambridge Univ. Press, 2007.

[22] A. Luis, "Degree of coherence for vectorial electromagnetic fields as the distance between correlation matrices," J. Opt. Soc. Amer. A, vol. 24, no. 4, pp. 1063-1068, 2007.

[23] M. Migliaccio and G. Ferrara, "A simple polarimetric field characterisation in reverberating chambers," IET Microw. Antennas Propag., vol. 1, no. 5, pp. 1029-1033, 2007.

[24] J. J. Gil, "Polarimetric characterization of light and media," Eur. Phys. J. Appl. Phys., vol. 40, no. 1, pp. 1-47, 2007.

[25] M. R. Dennis, "Geometric interpretation of the three-dimensional coherence matrix for non paraxial polarization," J. Opt. A: Pure Appl. Opt., vol. 6, no. 3, pp. S26-S31, 2004.

[26] J. J. Gil, J. M. Correas, P. A. Melero, and C. Ferreira, "Generalized polarization algebra," Monografías del Seminario Matemático García de Galdeano, vol. 31, pp. 161-167, 2004.

[27] J. J. Gil and I. San José, "3D polarimetric purity,” Opt. Commun., vol. 283 no. 22 , pp. 4430-4434, 2010.

[28] I. San José and J. J. Gil, "Invariant indices of polarimetric purity. Generalized indices of purity for $n \times n$ covariance matrices," Opt. Commun., vol. 284, no. 1, pp. 38-47, 2011.

[29] M. Migliaccio, J. J. Gil, A. Sorrentino, F. Nunziata, and G. Ferrara, "The polarization purity of the electromagnetic field in a reverberating chamber," IEEE Trans. Electromagn. Compat., vol. 58, no. 3, pp. 694-700, Jun. 2016.

[30] J. J. Gil, "Interpretation of the coherency matrix for three-dimensional polarization states," Phys. Rev. A, vol. 90, no. 4, pp. 1-11, 2014.

[31] C. Brosseau, "Evolution of the Stokes parameters in optically anisotropic media," Opt. Lett., vol. 20, no. 11, pp. 1221-1223, Jun. 1995.

[32] J. J. Gil, "Intrinsic Stokes parameters for 3D and 2D polarization states," J. Eur. Opt. Soc., vol. 10, pp. 15054-15058, 2015

[33] J. J. Gil and R. Ossikovski, Polarized Light and the Mueller Matrix Approach. Boca Raton, FL, USA: CRC Press, 2016.

[34] J. J. Gil, "Components of purity of a three-dimensional polarization state," J. Opt. Soc. Amer. A, vol. 33, no. 1, pp. 40-43, 2016.

[35] J. J. Gil, A. T. Friberg, T. Setälä and I. San José, "Structure of polarimetric purity of three-dimensional polarization states," Phys. Rev. A, vol. 95, no. 5, pp. 1-10, 2017.

[36] J. J. Gil, A. Norrman, A. T. Friberg, and T. Setälä, "Polarimetric purity and the concept of degree of polarization," Phys. Rev. A, vol. 97, no. 2, 2018 Art. no. 023838 .

[37] J. J. Gil, A. Norman, A. T. Friberg, and T. Setl, "Nonregularity of threedimensional polarization states," Opt. Lett., vol. 43, no. 19, pp. 4611-4614, 2018. 
[38] D. Senic et al., "Isotropy study for over-the-air measurements in a loaded reverberation chamber," in Proc. IEEE Int. Symp. Electromagn. Compat. Signal/Power Integrity, 2017, pp. 124-129.

[39] C. L. Holloway, D. A. Hill, J. M. Ladbury, P. F. Wilson, G. Koepke, and J. Coder, "On the use of reverberation chambers to simulate a Rician radio environment for the testing of wireless devices," IEEE Trans. Antennas Propag., vol. 54, no. 11, pp. 3167-3177, Nov. 2006.

[40] G. Ferrara, M. Migliaccio, and A. Sorrentino, "Characterization of GSM non-line-of-sight propagation channels generated in a reverberating chamber," IEEE Trans. Electromagn. Compat., vol. 49, no. 3, pp. 467-473, Aug. 2007.

[41] P. Corona, G. Latmiral, and E. Paolini, "Performance and analysis of reverberating enclosure with variable geometry," IEEE Trans. Electromagn. Compat., vol. EMC-22, no. 1, pp. 2-5, Feb. 1980.

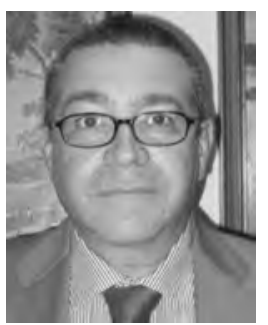

Maurizio Migliaccio (Fellow, IEEE) was born in Naples, Italy, in 1962. He received the Laurea (Hons.) degree in electronic engineering from the Università degli Studi di Napoli Federico II, Naples, Italy, in 1987.

He is currently a Full Professor of Electromagnetics with the Università degli Studi di Napoli Parthenope, Naples, Italy. He has authored or coauthored more than 100 peer-reviewed journal papers in applied electromagnetics.

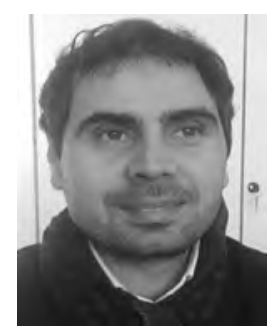

Antonio Sorrentino (Member, IEEE) was born in Italy. He received the M.Sc. degree (summa cum laude) in telecommunication engineering and the $\mathrm{Ph} . \mathrm{D}$. degree in electromagnetic fields from the University of Naples Parthenope, Naples, Italy, in 2005 and 2008, respectively.

Since 2008, he has been involved in electromagnetic laboratory applications. He has authored or coauthored more than 30 peer-reviewed journal papers in applied electromagnetics, including the reverberation chamber, polarimetic characterization of electromagnetic fields within reverberation chamber, and characterization of the wireless propagation channels.

Dr. Sorrentino is a member of the Scientific Committee of the IEEE Electromagnetic Compatibility Italy Chapter.

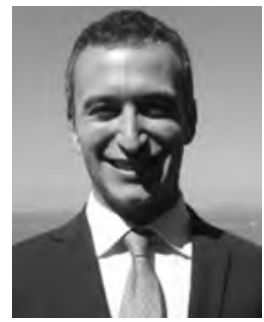

Ferdinando Nunziata (Senior Member, IEEE) was born in Avellino, Italy, in 1982. He received the Ph.D. degree in telecommunication engineering from the Università degli Studi di Napoli Parthenope, Naples, Italy, in 2008.

He has been an Associate Professor with the Università degli Studi di Napoli Parthenope since 2019. He has authored or coauthored more than 80 peerreviewed journal papers in applied electromagnetics, including the reverberation chamber, polarimetic characterization of electromagnetic fields within reverberation chamber, and characterization of the wireless propagation channels.

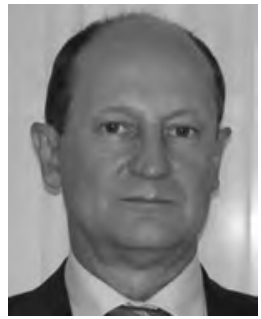

José Jorge Gil received the Ph.D. degree in physics from the University of Zaragoza, Zaragoza, Spain, in 1983.

$\mathrm{He}$ has been a Professor with the University of Zaragoza since 1987. He was also the General Manager with the R\&D Department of the Spanish Company BGL from 1991 to 1996, where he led the development of wireless systems for interactive meetings, which deserved the Tecnova Award from the Spanish Industry Ministry in 1993. He has authored a large number of scientific articles and reports, including the book Polarized Light and the Mueller Matrix Approach (CRC Press, 2016). Prof. Gil was the recipient of the G. G. Stokes Award 2013 from the International Society for Optics and Photonics.

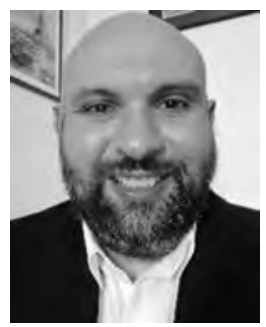

Sergio Cappa (Student Member, IEEE) was born in Naples, Italy, in 1984. He received the M.Sc. degree in telecommunication engineering and the Ph.D. degree in electromagnetic fields from the University of Naples Parthenope, Naples, Italy, in 2014 and 2020, respectively.

He has authored or coauthored more than ten scientific papers appeared in peer-reviewed journals and refereed international conferences. His current research interests include the reverberation chamber, polarimetic characterization of electromagnetic fields within reverberation chamber, and characterization of the wireless propagation channels in controlled environments. 